\title{
Editorial
}

\section{Evidence Based Management of COVID-19 - Bangladesh Perspective}

\author{
Md. Asadul Kabir
}

\begin{abstract}
(c) (1) (2)
DOI: https://doi.org/10.3329/jom.v23il.57929

Copyright: (C) 2022 Kabir A. This is an open access article published under the Creative Commons Attribution-NonCommercial-NoDerivatives 4.0 International License, which permits use, distribution and reproduction in any medium, provided the original work is properly cited, is not changed in any way and it is not used for commercial purposes.
\end{abstract}

Received: 10 October, 2021;

Accepted: 14 November 2021

\section{Introduction}

The World Health Organization (WHO) declared coronavirus disease 2019 (COVID-19) as Public Health Emergency of International Concern leading to a state of pandemic, on getting alerts from Wuhan, China. COVID-19, recently evolved respiratory disease of potential virulence, is caused by novel severe acute respiratory syndrome coronavirus 2 (SARS-CoV-2) that has come to acquire the status of an abundant global pandemic affecting $\mathbf{2 7 5 , 1 0 2 , 4 2 8}$ all over the world. In Bangladesh, 1,581,343 individuals are affected, 28,050 died till last $20^{\text {th }}$ December,2021. ${ }^{1}$ It is speculated that the epidemiology and clinical presentation could be different from elsewhere and might influence the prognosis of disease. Multiple social factors such as health awareness, over-crowding, personal hygiene, undiagnosed comorbid condition leads to variation in rate of COVID-19 infection. More than $75 \%$ of patients hospitalized with COVID-19 require supplemental oxygen therapy. For patients who are unresponsive to conventional oxygen therapy, may require heated high-flow nasal canula oxygen or invasive mechanical ventilation. Evidence based guideline initiatives have been established by many countries and professional societies, including guidelines that are updated regularly by the Director General Health Services.

\section{Pathophysiology of COVID-19}

Coronaviruses are large, enveloped, single-stranded RNA viruses found in humans and other mammals, such as dogs, cats, chicken, cattle, pigs, and birds. Coronaviruses cause respiratory, gastrointestinal, and neurological disease, thrombotic complications, such as deep venous thrombosis,

Corresponding author: Prof. Md. Asadul Kabir, Professor, Department of Medicine, Dhaka Medical College, Dhaka. E-mail: chapalk40@gmail.com pulmonary embolism, and thrombotic arterial complications (e.g. limb ischemia, ischemic stroke, myocardial infarction) in critically ill patients. The development of viral sepsis, defined as life threatening organ dysfunction caused by a dysregulated host response to infection, may further contribute to multi organ failure. The clinical spectra of COVID-19 is varied, ranging from mild-to-moderate symptoms of cough, sore throat, headache, rhinorrhea, vomiting, diarrhea, fever, shortness of breath along with signs and symptoms complex of severe pneumonia, acute respiratory distress syndrome, septic shock, and/or multiple organ failure. ${ }^{2}$ About $80 \%$ of the individuals with SARSCoV-2 either remain asymptomatic or show mild symptoms ; these may be managed at home or in isolation centers to check the spread of transmission. The remaining $10-15 \%$ have moderate to severe symptoms, and need institutional care ranging from oxygen therapy, intensive care to ventilator support. The elderly and those with comorbidities (e.g., diabetes, hypertension, coronary heart disease, chronic obstructive lung disease, renal diseases, etc.) are at higher risk of developing florid symptoms and meet adverse outcomes. ${ }^{3}$

\section{Various trials of drugs and WHO recommendation}

SARS-CoV-2 rapidly spread from China until it was defined a pandemic by WHO in March 2020. Related scientific papers have rapidly extended information regarding the diagnosis, treatment and epidemiology of COVID-19 infection. To date, no definitive treatment is available to defeat the virus and therapies are mainly based on existing drugs used to treat other conditions. Existing therapies used in several clinical trials work by affecting the biology of COVID-19 and/or counteracting the harmful host excessive immune response. About 526 ongoing clinical trials for COVID-19 to provide a 


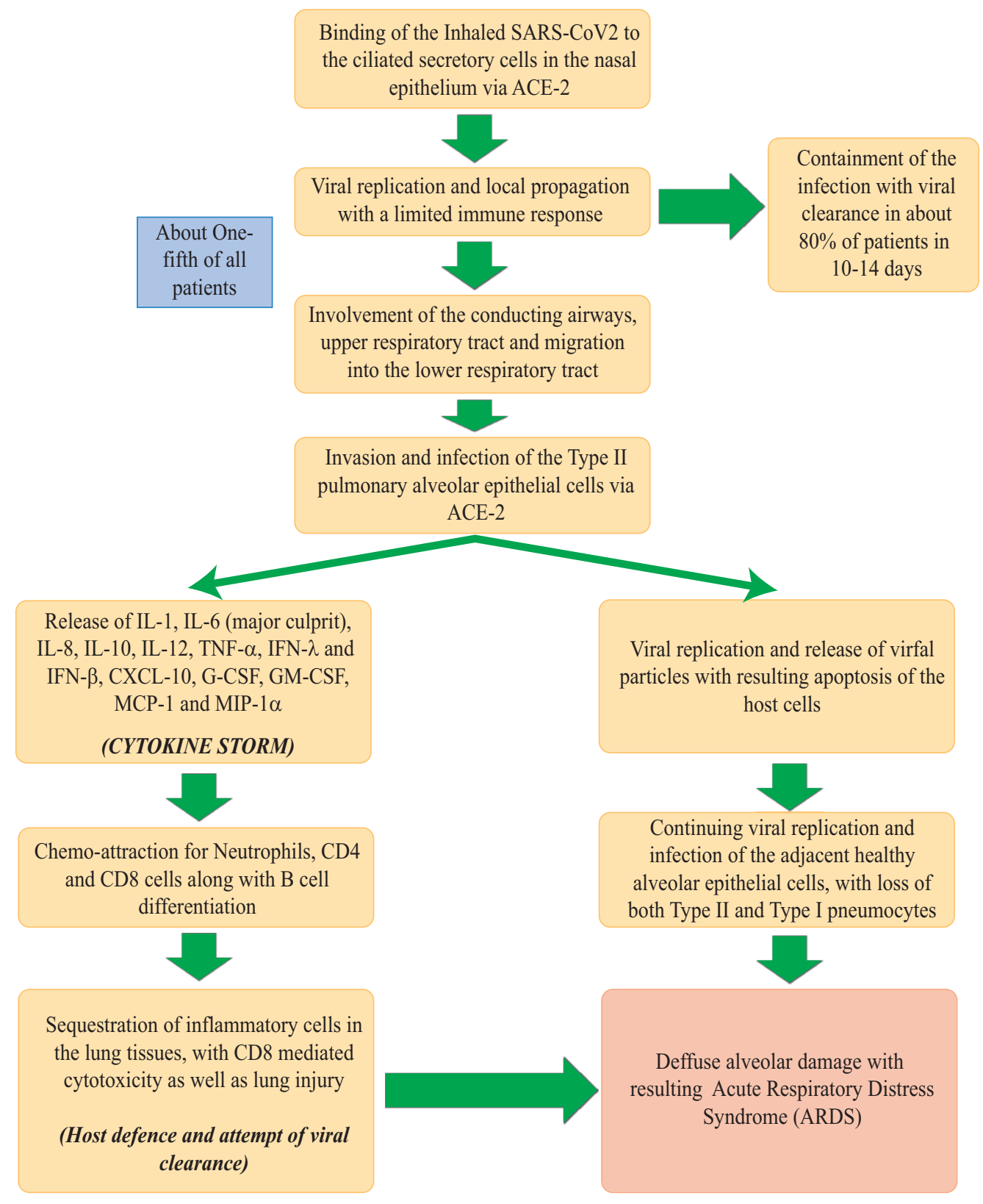

Figure: Pathophysiology of COVID-19. ${ }^{4}$

perspective on the first 6 months of global efforts to identify an effective therapy. The drugs most actively tested in various centers include hydroxychloroquine, ritonavir, azithromycin, lopinavir chloroquine and ivermectin, antivirals (eg, remdesivir, favipiravir), antibodies (eg, convalescent plasma, hyperimmune immunoglobulins), anti-inflammatory agents (dexamethasone, statins), targeted immunomodulatory therapies (eg, tocilizumab, sarilumab, anakinra, ruxolitinib), anticoagulants (eg, heparin), and antifibrotics (eg, tyrosine kinase inhibitors). It is likely that different treatment modalities might have different efficacies at different stages of illness and in different manifestations of disease. Viral inhibition would be expected to be most effective early in infection, while, in hospitalized patients, immunomodulatory agents may be useful to prevent disease progression and anticoagulants may be useful to prevent thromboembolic complications. Till date 5372 studies on COVID-19 are registered in WHO database mostly related to treatment and vaccine. Randomized clinical trials are ongoing and should provide more guidance. ${ }^{5}$

The largest WHO COVID-19 solidarity therapeutic clinical trial is going on involving 14,200 hospitalized patients of 600 hospitals of 52 countries by 2000 researchers. This large 
global randomized control trial is designed to give robust results on whether a drug can save lives in those hospitalized with severe or critical COVID-19. The WHO Solidarity PLUS Trial will initially evaluate three treatment arms: artesunate, infliximab and imatinib, in addition to the local standard of care. ${ }^{6}$ The Recovery trial shows patients hospitalized with Covid-19, the use of dexamethasone resulted in lower 28day mortality among those who were receiving either invasive mechanical ventilation or oxygen alone at randomization but not among those receiving no respiratory support. ${ }^{7}$ Adaptive COVID-19 Treatment Trial (ACTT-1) show that remdesivir was superior to placebo in shortening the time to recovery in adults who were hospitalized with Covid-19 and had evidence of lower respiratory tract infection. ${ }^{8}$

The evidence base for therapeutics for COVID-19 is increasing rapidly, and some treatments of proven benefit have emerged. Numerous randomized trials of many drugs are underway to further inform practice. The latest version of the WHO living guideline contains new recommendations on a combination of neutralizing monoclonal antibodies (casirivimab and imdevimab) based on four randomized controlled trials (RCTs). In this update, the panel makes a conditional recommendation to use casirivimab and imdevimab in non-severe patients, the condition being patients' risk of severe disease: patients at highest risk represent good candidates for use of the intervention. ${ }^{9}$ Previous recommendations include:

- a strong recommendation for systemic corticosteroids in patients with severe and critical COVID-19;

- a strong recommendation for IL-6 receptor blockers (tocilizumab or sarilumab) in patients with severe and critical COVID-19;

- a conditional recommendation against systemic corticosteroids in patients with non-severe COVID-19;

- a conditional recommendation against remdesivir in hospitalized patients with COVID-19;

- a strong recommendation against hydroxychloroquine in patients with COVID-19 of any severity;

- a strong recommendation against lopinavir/ritonavir in patients with COVID-19 of any severity;

- a recommendation against ivermectin in patients with COVID-19 of any severity, except in the context of a clinical trial. ${ }^{9}$

\section{Management of COVID-19 in Bangladesh}

Bangladesh, a country of 170 million people, detected its first case of COVID-19 on 8th March 2020 and the first fatality was recorded on 18th March 2020. The numbers of patients with morbidity and fatality due to COVID-19 have been increasing since then and about 20-25\% tested for SARSCoV-2 are positive for the virus and many of these patients need hospitalization. The management of patients with COVID-19 remains a challenge for Bangladesh. Despite being a developing country, Bangladesh has taken some vital steps in the health sector. Significantly, the reduction of infant mortality, maternal care, and immunization are the key steps to ensure healthcare for all. However, Bangladesh, one of the most populous countries in the world with a density of 1104 persons per sq. $\mathrm{km}$, is experiencing quick spread of COVID-19 after the first case was identified. Large scale testing allows health services to quickly identify who has the disease and arrange for them to receive the care needed. Effective testing programs around the world allow governments and health authorities to understand how prevalent the disease is and how it is evolving.

More than 39 hospitals all over the country are directly involved in COVID-19 management, including Dhaka Medical College Hospital, Kurmitola General Hospital, Mugda Medical College and Hospital, Kuwait Bangladesh Friendship Government Hospital, Bangabandhu Sheikh Mujib Medical University (BSMMU), Combined Military Hospital(CMH). Private hospitals were initially not oriented with COVID-19 management, but later on they came forward. Most of the centers follow national guidelines on clinical management of COVID-19 which is prepared and regularly updated by Bangladesh Society of Medicine. Despite WHO and our national guideline recommendations, it has been reported that azithromycin is widely used with other drugs such as dexamethasone, remdesivir, baricitinib, oseltamivir and tocilizumab without proper indication in different hospitals of Bangladesh. Not only azithromycin, injudicious use of other broad spectrum antibiotics was reported. Antibiotic use appears to be high among COVID-19 patients with one report indicating up to $45 \%$ of coronavirus patients were receiving antibiotic treatment. ${ }^{10}$

It is evidenced that increased utilization of antibiotics (70.6\%), analgesics (97.6\%), vitamins (90.6\%) in Bangladesh over last two years. In initial phase of COVID-19 pandemic, random use of hydroxychloroquine, ivermectin, doxycycline was observed in different institutions as well as in mass population as OTC drug. ${ }^{11}$ It is the consequence of lack central monitoring system, some centers of Bangladesh did not follow our national guideline, rather help to implement over drug use without proper indication.

\section{Outcome following irrational treatment}

The widespread off label and irrational use of drugs for Covid-19 is cause for deep concern and might be contributing 
to the overall morbidity and mortality that has been attributed primarily to the infection. Of particular concern are the use of macrolide antibiotics for a viral infection, and the use of antimalarial drugs CQ and HCQ for prophylaxis and treatment of mild Covid-19. There is already enough evidence from observational and clinical studies to show that risks of serious adverse events clearly outweigh hypothetical clinical benefits when these antimalarials are used for preventive interventions and treatment of mild to moderate Covid-19. ${ }^{12}$

Antimicrobial resistance is one of the biggest challenges facing modern medicine. Because the management of COVID-19 is increasingly becoming dependent on pharmacological interventions, there is greater risk for accelerating the evolution and spread of antimicrobial resistance. Clinically-unjustified use of antibiotics is thought to be linked with antimicrobial resistance, which is estimated to claim death of 700,000 people per annum. ${ }^{13}$

With hospitalizations exceeding normal capacity due to COVID-19 combined with impaired immune function in patients, risks of co-infections have substantially increased. The gap in a comprehensive understanding of coinfection and comorbidity caused by COVID-19 has led to rapidly changing protocols for patient handling, including administering multiple drugs around the world. The use of antiparasitic, antiviral, antibacterial, and anti-inflammatory drugs for preventing secondary infections in patients with COVID-19 during a prolonged pandemic will inevitably invite future complications, including aggravation of antimicrobial resistance. This is particularly relevant in light of the successive emergence of mutations that increase SARS-CoV2 fitness, which could be responsible for recurrent COVID19 waves. Of note, because most of these drugs are used for other target pathogens, we might not only increase resistance in COVID-19 but also face challenges in the treatment of other bacterial and viral infections.

\section{Conclusion}

It is true that the recent emergence of the Omicron variant has contributed to increasing COVID-19 cases rapidly in whole world. But we must be vigilant and cautious that the fight against COVID-19 does not invite another significant threat to humankind. As new evidence emerges, it is imperative that current and potential treatment options are frequently re-evaluated in order to offer the best possible care under such unprecedented circumstances. Many arguments regarding EBM in pandemic-like situations must use different evidentiary standards. The hierarchies of evidence that emphasize its scientific quality are still relevant and every center of our country should manage their patients on the basis of evidence, not mythical.

\section{References}

1. The Real Time Statistics Project. Available from: http// www.worldometers.info. [Last accessed on 2021 December 20]

2. Huang C, Wang Y, Li X, Ren L, Zhao J, Hu Y, et al. Clinical features of patients infected with 2019 novel coronavirus in Wuhan, China. Lancet. 2020;395:497-506.

3. Guan WJ, Liang WH, Zhao Y, Liang HR, Chen ZS, Li YM, et al. Comorbidity and its impact on 1590 patients with COVID-19 in China: A nationwide analysis. Eur Respir J 2020;55:2000547.

4. Anant Parasher, COVID-19: Current understanding of its Pathophysiology, Clinical presentation and Treatment; BMJ Journal; 2020 sep 25; 97(1147)

5. https://clinicaltrials.gov/ct2/who_table

6. https://www.who.int/emergencies/diseases/novelcoronavirus-2019/global-research-on-novel-coronavirus2019-ncov/solidarity-clinical-trial-for-covid-19-treatments.

7. The RECOVERY Collaborative Group. Dexamethasone in Hospitalized Patients with COVID-19 The RECOVERY Collaborative Group; NEJM; 2021 Feb 25: 384.

8. John H. Beigel, Kay M. Tomashek, Lori E. Dodd, Ph, Aneesh K. Mehta, Barry S. Zingman, Andre C. Kalil, et al., for the ACTT-1 Study Group Members. Remdesivir for the Treatment of Covid-19 — Final Report; NEJM; 2020 Nov 5: 383

9. Living guidance for clinical management of COVID-19. World Health Organization. $3^{\text {rd }}$ ed. 23 Nov 2021.

10. Abul KalamI, Shahanaj Shano, Mohammad Asif Khan, Ariful Islam, Narelle Warren, Mohammad Mahmudul Hassan, Mark Davis; Understanding the social drivers of antibiotic use during COVID-19 in Bangladesh: Implications for reduction of antimicrobial resistance; Research Article; 2021 Dec $14 ; 16(12)$

11. Mainul Haque, Salequl Islam, Samiul Iqbal, Umme Laila Urmi, Zubair Mahmood Kamal, Aminur Rahman; Availability and price changes of potential medicines and equipment for the prevention and treatment of COVID-19 among pharmacy and drug stores in Bangladesh; findings and implications; Bangladesh Journal of Medical Science; 2020 July 10: Vol 19: 'Special issue on Covid 19' 2020.

12. Francisco Jose Roma Paumgartten; Off label, compassionate and irrational use of medicines in Covid-19 pandemic, health consequences and ethical issues; ResearchGate, Sep 20.

13. Murray AK. The Novel Coronavirus COVID-19 Outbreak: Global Implications for Antimicrobial Resistance. Front Microbiol. 2020; 11: 1-4. 11. 